\title{
Episodic traces and statistical regularities: Paired associate learning in typical and dyslexic readers
}

Short title: Paired associate learning in typical and dyslexic readers

Manon Wyn Jones ${ }^{1}$, Jan-Rouke Kuipers ${ }^{2}$, Sinead Nugent ${ }^{1}$, Angelina Miley ${ }^{1}$, and Gary Oppenheim ${ }^{13}$

${ }^{1}$ School of Psychology, Bangor University, Bangor, Wales. LL57 2AS.

${ }^{2}$ Psychology: Faculty of Natural Sciences, University of Stirling, Stirling, Scotland. FK9 4LA.

${ }^{3}$ Department of Psychology, Rice University, Houston, TX, USA.

Address for correspondence:

Dr. Manon Jones,

School of Psychology

Bangor University

Brigantia Building

Penrallt Road

Bangor

LL57 2AS

Tel: 01248382319

Email: manon.jones@bangor.ac.uk

Accepted refereed manuscript of: Jones MW, Kuipers JR, Nugent S, Miley A \& Oppenheim G (2018) Episodic traces and statistical regularities: Paired associate learning in typical and dyslexic readers, Cognition, 177, pp. 214-225.

DOI: $\underline{10.1016 / j . c o g n i t i o n .2018 .04 .010}$

(C) 2018, Elsevier. Licensed under the Creative Commons Attribution-NonCommercial-

NoDerivatives 4.0 International http://creativecommons.org/licenses/by-nc-nd/4.0/ 


\begin{abstract}
Learning visual-phonological associations is a key skill underlying successful reading acquisition. However, we are yet to understand the cognitive mechanisms that enable efficient learning in good readers, and those which are aberrant in individuals with developmental dyslexia. Here, we use a repeated cued-recall task to examine how typical and reading-impaired adults acquire novel associations between visual and phonological stimuli, incorporating a looking-at-nothing paradigm to probe implicit memory for target locations. Cued recall accuracy revealed that typical readers' recall of novel phonological associates was better than dyslexic readers' recall, and it also improved more with repetition. Eye fixation-contingent error analyses suggest that typical readers' greater improvement from repetition reflects their more robust encoding and/or retrieval of each instance in which a given pair was presented: whereas dyslexic readers tended to recall a phonological target better when fixating its most recent location, typical readers showed this pattern more strongly when the target location was consistent across multiple trials. Thus, typical readers' greater success in reading acquisition may derive from their better use of statistical contingencies to identify consistent stimulus features across multiple exposures. We discuss these findings in relation to the role of implicit memory in forming new visual-phonological associations as a foundational skill in reading, and areas of weakness in developmental dyslexia.
\end{abstract}

Keywords: Paired associated learning; Visual-phonological binding; Developmental dyslexia; Eyetracking; Looking-at-nothing; Statistical learning; Episodic memory

\title{
Introduction
}

Converting letters into sounds is a fundamental skill in reading acquisition, explaining both clinical and sub-clinical individual differences in reading abilities. Poor visual-phonological mapping is a 
defining feature of developmental dyslexia (Warmington \& Hulme, 2012; Wimmer, 1993; Lervag \& Hulme, 2010), and an emerging body of research suggests that competence in forming novel visual-phonological associations provides a strong, unique predictor of reading ability among typical readers as well (Ehri, 2005; Ehri \& Saltmarch, 1995; Wang, Allen, Lee, \& Hsieh, 2015). Yet, despite repeated demonstrations of visual-phonological mapping skills as an important explanatory variable in reading, the cognitive mechanisms underlying them remain largely unknown. In this paper, we consider the contributions of episodic memory and statistical learning to typical and dyslexic adult readers' acquisition of new visual-phonological associations over multiple exposures.

\section{Learning new visual-phonological associations}

In the domain of reading, learning visual-phonological associations can be considered the cornerstone of letter-sound acquisition, and is duly instantiated in connectionist models of orthographically driven phonological retrieval (Harm \& Seidenberg, 1999; Manis, Seidenberg, \& Doi, 1999; Seidenberg \& McClelland, 1989). Seidenberg and McClelland's (1989) model, for instance, characterises skilled reading as a mapping from letters to phonological forms, gradually acquired via a backpropogation algorithm that is best understood as implementing implicit or statistical learning. Implicit learning can also leverage explicit memory (e.g. McClelland, McNaughton, O’Reilly, 1995), a relationship often emphasised by phonological-awareness-based approaches to reading instruction (e.g. Seidenberg, 2017).

One method that researchers have used to examine the relationship between novel visual-phonological mapping and reading acquisition is paired associate learning (cf. Vellutino, Steger, Harding, \& Phillips 1975; Hulme, Goetz, Gooch, Adams, \& Snowling, 2007; Wang, Wass, \& Castles, 2016). This method uses explicit cued recall (e.g. "Which word goes with this picture/shape?") to probe participants' gradual acquisition of associations between arbitrarily 
paired stimuli, typically over the course of four or five repetitions. Recall of unimodal associations (e.g., a visual object paired with another visual object or a sound paired with another sound) is typically used to establish a baseline, whereas recall of cross-modal associations (e.g., a visual object paired with an auditory stimulus), is assumed to more directly reflect the cross-modal skills necessary for learning to read. It has recently been claimed that only cross-modal association recall contributes unique variance to reading abilities, as evidenced in measures such as exception word reading, nonword decoding, and reading speed (Hulme et al., 2007; Warmington \& Hulme, 2012), perhaps because readers specifically use their visual-phonological paired-associate learning abilities in forming orthographic knowledge (Wang et al., 2016). For more general reading measures, particularly those relying more on a verbal component, the cross-modal aspect of pairedassociate learning is less important than the verbal component per se (Litt, de Jong, van Bergen, \& Nation, 2013; Litt \& Nation, 2014).

As well as predicting variation in reading skill among typical readers, paired-associate learning performance discriminates dyslexic from typically developing children and adults: individuals with dyslexia typically recall associated representations much less accurately (Jones, Parra, Branigan, \& Logie, 2013; Litt \& Nation, 2014; Messbauer \& de Jong, 2003; Vellutino, Scanlon, \& Spearing, 1995; Wimmer, Mayriner, \& Landerl, 1998). If visual-phonological association learning is impaired in dyslexia, what then are the cognitive mechanisms that allow skilled readers to form stable associations where those with dyslexia cannot? Because pairedassociate learning in reading research is typically considered an index of a reading-related subskill—without explicit reference to specific underlying cognitive processes-we suggest that resituating it within a working memory framework may help delineate mechanisms under lying success and failure in this skill.

\section{Learning mechanisms}


Beyond paired-associate learning — reflecting early acquisition processes—visualphonological associations remain impaired in dyslexia, even for highly-overlearned pairings, such as letter-to-letter sound associations (Blau, Van Atteveldt, Ekkebus, Goebel, \& Blomert, 2009; Jones, Kuipers, \& Thierry, 2016; Žarić et al., 2015). Failure to learn efficiently during the first exposures must therefore have long-term consequences for memory consolidation, despite potentially ameliorating factors such as practice and maturation (Snowling, 2000). What then are the cognitive mechanisms that allow skilled readers to form stable associations where readers with dyslexia cannot?

In the working memory literature, forming visual-phonological associations can be considered a subtype of binding, that is, integrating individual features to create a compound representation that can be retrieved as a single unit (Brockmole \& Franconeri, 2009). Although associations can eventually be encoded into long term memory, establishing a novel binding — such as a new visual/phonological pairing_requires maintenance in a capacity-limited episodic buffer (Baddeley, Allen, \& Hitch, 2011), placing significant demands on attentional resources (Hommel \& Colzano, 2009; Vanrullen, 2009). Novel bindings also crucially depend on spatial-temporal proximity for detecting and recalling associations (Logie, Brockmole, \& Jaswall, 2011; Treisman, 2006; Treisman \& Gelade, 1980; Treisman \& Zhang, 2006).

Episodic encoding and retrieval of such spatial-temporal proximities has been cited as a basis for a "looking-at-nothing" (LAN) phenomenon, in which verbally recalling auditory information is associated with looks to previously relevant screen locations (Ferreira, Apel, \& Henderson, 2008; Hoover \& Richardson, 2008; Jahn \& Braatz, 2014; Laeng et al., 2014; Richardson \& Spivey, 2000; Scholz, Mehlhorn, \& Krams, 2016; but see Staudte \& Altmann, 2017). Due to the overlap in processes engaged in the encoding and retrieval of an event stored in episodic memory, activating spatial information may spread to the oculomotor programme conducted during encoding, which then triggers associated visual and/or auditory components learned during encoding (Johansson et 
al., 2012; Johansson \& Johansson, 2014; Laeng et al., 2014). Perhaps the strongest functional interpretation of looking-at-nothing claims a relationship between eye movements and verbal recall, in which stronger tendencies to look-at-nothing are associated with increased accuracy (Scholz et al., 2016; Wantz et al., 2016).

Recalling features of episodic memories, such as the spatial configuration of an item display during encoding, may therefore involve re-binding multimodal (e.g., visual-phonological) representations. This rebinding may be an important ability underlying reading acquisition, as evidenced by its impairment in readers with dyslexia: we have previously found that, following a single exposure, adult typical readers recall visual-phonological pairs more accurately than dyslexic readers, but this difference only emerges in the presence of spatial cues (Jones, Branigan, Parra and Logie, 2013).

Fluent reading involves automatizing access to visual-phonological associations (Froyen, Bonte, van Atteveldt, \& Blomert, 2009; Froyen, Willems \& Blomert, 2011; Jones, Kuipers, \& Thierry, 2016). Thus, episodic contributions to visual-phonological mappings must gradually decrease as repeated presentations of letter-sound correspondences strengthen implicit pathways for reading (LaBerg and Samuels, 1974; Denckla \& Rudel, 1976; Jones, Ashby, \& Branigan, 2013; Jones, Obregon, Kelly, \& Branigan, 2008). We might therefore expect such episodic memorybased effects to decrease with practice. Here, it may be useful to consider letter learning as an example of the more general process of category learning: skilled reading would be impossible without identifying each printed letter (e.g. b, $b$, or B) as an instance of its more general letter category ('b'), inheriting learned knowledge about that category (that it maps to the sound /b/). Exemplar-based theories of category learning (e.g. Hintzman, 1986; Medin \& Schaffer, 1978; Nosofsky, 1986), describe a process whereby each instance of a stimulus is stored in memory and contributes to category development: as learning progresses, category use becomes less dependent on the details of any particular instance (e.g., Logan, 2002; Krushke, 1992; Love, Medin, \& 
Gureckis, 2004). Implicit or statistical learning can be thought of as the process of developing these more 'abstracted' representations (see also Altmann, 2017).

Indeed, the ability to track simple statistics, such as sensitivity to repeated stimuli and stimulus sequences is a strong predictor of reading ability (cf. Ahissar, 2007). For instance, implicit memory for previous exposures to perceptual stimuli has been shown to decay more quickly in dyslexic readers compared with typical readers (Jaffe-Dax, Raviv, Jacoby, Loewenstein, \& Ahissar, 2015; Jaffe-Dax, Lieder, Biron \& Ahissar, 2016; Jaffe-Dax, Frenker, \& Ahissar, 2017). Such decay may reflect dyslexic readers' failure to adequately encode previous instances of a given stimulus, leading to 'noisy' or ineffective processing of the current instance (ibid). Although this explanation has primarily been applied to extracting central tendencies from perceptual instances, it seems plausible that poor encoding of individual instances could similarly affect processes such as the gradual automatization of access to bound visual-phonological representations.

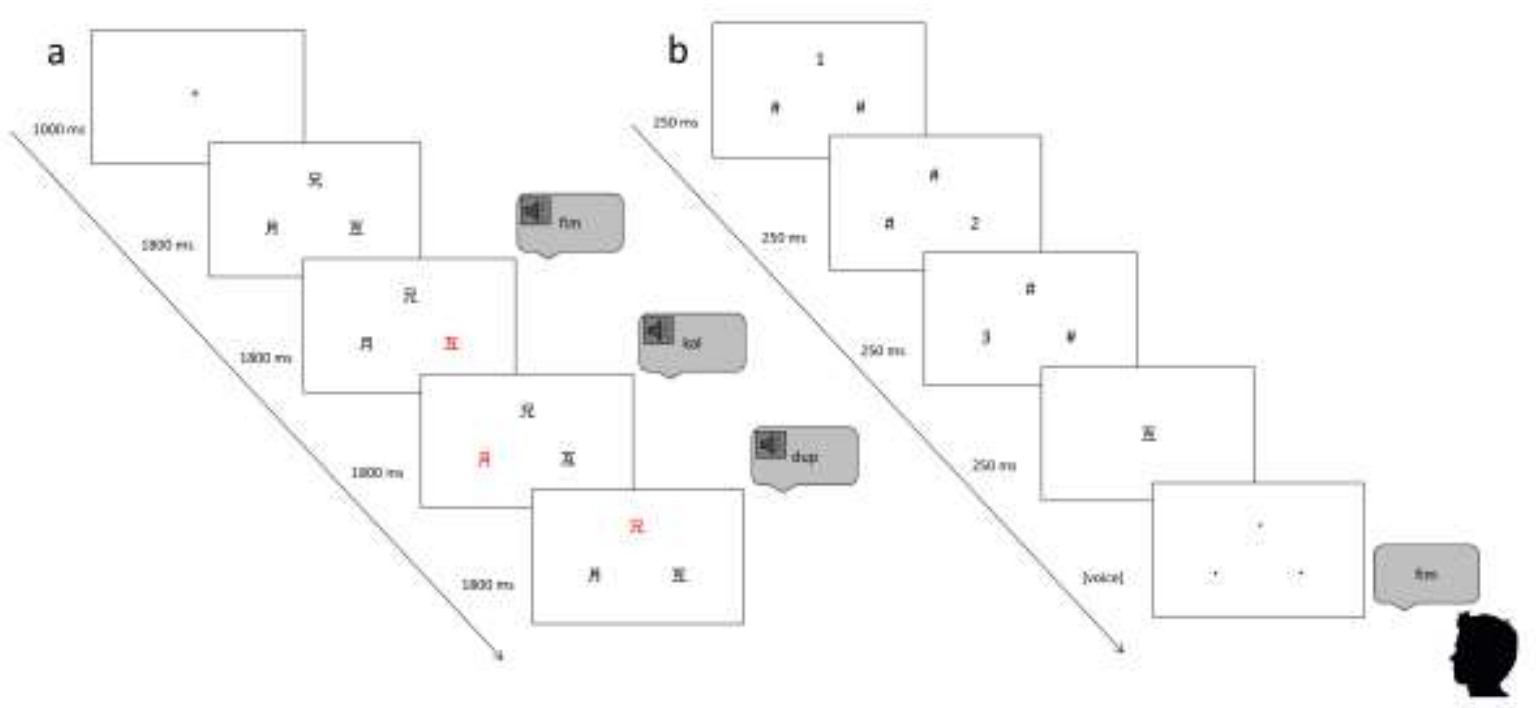

Figure 1: Each trial consisted of an encoding phase (Panel A) followed, after a 1000ms blank screen, by backward masking and a cued recall phase (Panel B). Encoding presented a set of Mandarin characters and nonsense syllables; numbers and hash symbols, then masked their locations, to minimize iconic memory and discourage rehearsal. Then probe and recall screens cued the participant to orally recall the associated nonword. Onscreen fixations were recorded only during this final recall screen.

\section{The current study}


In the above, we have described a view of reading acquisition wherein skilled reading development involves a transition from an initial stage, in which reading depends on recalling visualphonological bindings as presented in individual episodes, to later stages, in which it depends more on integrated mappings developed through repetition, that is, shifting from recalling a specific instance to recalling statistical tendencies. Extant literature suggests that dyslexic readers experience difficulty with both.

The current study therefore directly compares typical and dyslexic readers' acquisition of new shape-nonword pairs, via a paired-associate learning paradigm in which we track cued recall accuracy as a function of stimulus-pair repetition and 'looks at nothing'. Participants encounter 30 novel shape-nonword pairs as they appear 18 times over the course of the experiment. On each trial, three shapes appear, highlighted in turn as their associated nonword is played over loudspeakers (Figure 1a, see Methods section; as in Jones et al, 2013); they then disappear, and one is cued for recall (Figure 1b). Each shape is consistently and uniquely bound to a single nonword, but appears in each of three on-screen locations with equal probability; thus participants should gradually distinguish between the shape-sound binding as a consistent association, and its spatial location as an inconsistent feature bound to particular episodes. Tracking 'looks to nothing' during recall thus provides an index of episodic contributions to visual-phonological association retrieval. This structure allows us to test, in groups of typical and dyslexic readers: 1) how memory for recent episodic detail is associated with accurate recall of visual-phonological bindings, and 2) how recall of phonological and spatial features changes as a function of repeated exposure.

Previous empirical findings afford the general expectation that typical readers should recall visual-phonological bindings better than dyslexic readers (e.g. Hulme et al., 2007; Messbauer \& deJong, 2003; Jones et al., 2013). Given that dyslexic readers fail to automate visual-phonological connections in the longer term, we also expect typical readers to benefit more from repetition. More 
interesting is the question of how these patterns would be associated with changes in the recruitment of episodic detail, as indexed by looks-to-nothing.

In general, although looking-at-nothing is typically interpreted as a marker of successful recall (Altmann, 2004; Ferreira et al., 2008), there is also evidence that the behaviour decreases with repetition (Scholz et al., 2011, 2016; Wantz, Martarelli, \& Mast, 2016), suggesting a reduction in the use of inconsistent episode-bound memory details. In the current experiment, we would therefore expect relevant looks to nothing to similarly decrease as visual-phonological pairs are repeatedly presented in multiple locations.

Whilst previous studies have reported a benefit associated with looks to previous target locations, relative to a non-fixation baseline, including distractors in our paradigm allows us to assess the possible cost of activating incorrect spatial information, i.e. that associated with a competitor stimulus. Given previous findings, we also expect that specifically fixating a targetrelevant location - rather than a non-target-relevant ('distractor') location-should be associated with better recall of other concept components (Scholz et al., 2016), at least for typical readers. To the extent that phonological retrieval involves specifically recalling the most recent episode, fixations to a target's most recent location (i.e., that of the current trial) should be associated with more accurate phonological recall. And, to the extent that participants form a longer-term representation of the visual-phonological binding, engaging statistical learning to incorporate previous instances, recall accuracy should also be modulated by fixations to a target's other previous locations.

How might dyslexic readers' fixation patterns differ? Recall that dyslexics generally have worse recall for such bindings and benefit less from repetition. If such difficulties reflect failure to encode or re-activate location information (Jones et al., 2013), then dyslexics may be less likely to fixate target-relevant locations in the first place, and 'correct' fixations may be less strongly yoked to correct phonological recall. To the extent that dyslexics' episodic recall specifically omits spatial 
information, their fixations to a target's most recent location (i.e., that of the current trial) should be less associated with more accurate phonological recall. And, to the extent that dyslexic impairments reflect impaired statistical learning (Jaffe-Dax et al., 2015, 2017), their recall accuracy may also be less strongly modulated by fixations to a target's other previous locations.

\section{Methods}

Participants. Two groups of native British-English speaking students were recruited: 20 "typical readers" (age: $M=21.8, S D=1.82 ; 9$ females) and 20 "dyslexic" (age: $M=23.0, S D=3.04 ; 12$ females). The typical readers reported no difficulties associated with literacy, whilst members of the dyslexic group had been formally assessed by an Educational Psychologist during primary or secondary education. All participants had normal or corrected-to-normal vision and reported no other problems (e.g., hearing loss, specific language impairment, Attention Deficit Hyperactivity Disorder (ADHD) etc.). The study was approved by the Bangor University Ethics Committee and participants received course credit or payment for participation.

Literacy and General Cognitive Ability. Participants' allocation to reading groups was validated via a battery of six short tests: 1.) word reading efficiency and 2.) phonemic decoding efficiency subscales of the Test of Word Reading Efficiency (TOWRE, Torgesen et al., 1999); 3.) vocabulary (verbal) and 4.) matrix reasoning (nonverbal) indices of intelligence quotient (IQ) from the Wechsler Abbreviated Scale of Intelligence (WASI, Wechsler, 1999); and 5.) digit and 6.) letter versions of the rapid automatized naming (RAN) task from the Comprehensive Test of Phonological Processing (CTOPP; Wagner et al., 1999). 
Stimuli, Design and Procedure. Figure 1 depicts the process in each trial. On each trial, a participant saw three visual stimuli (Mandarin Chinese characters), presented in black on a highresolution LCD 40" screen (white background). Regions of interest (ROIs) for the eyetracking analyses each subtended a visual angle of $17^{\circ}$ (screen distance: $120 \mathrm{~cm}$ ), and were positioned according to the points of an equilateral triangle, in which the centre of the triangle was also the centre of the screen (see Figure 1a). Within each ROI, each character subtended a position of $11^{\circ}$ visual angle. Each character changed color to red, in turn, as its corresponding CVC nonword label was auditorily presented via loudspeakers $(44 \mathrm{kHz})$. Importantly, each character was paired with exactly one nonword (and vice versa) throughout the entire session, as in standard paired associate learning tasks, thus allowing us to assess the development of stable shape-sound bindings over multiple repetitions; the characters location within the stimulus array, however, was randomly assigned in each trial. The highlighting of each character / presentation of each nonword was followed immediately by presentation of the next character / nonword. After a 1000-ms blank screen, the participant saw an array in which two characters were replaced by hash symbols, and one character by a number. The numbers 1,2, 3 cycled through the three positions (see Figure 1b). These masking arrays served to suppress rehearsal of information in iconic memory. Finally, $1750 \mathrm{~ms}$ after the offset of the encoding-phase character screen, one character appeared in the centre of the screen for $250 \mathrm{~ms}$ as a recall probe. This probe was followed immediately by a 'recall screen': an array in which each character had been replaced by a small black circle $\left(0.25^{\circ}\right.$ of visual angle). Whilst viewing this array, the participant attempted to orally recall the nonword corresponding to the visual character probe. A voice-activated relay recorded the participant's vocal response latency, and the experimenter scored accuracy online. The experimenter's buttonpress response then terminated the trial.

Participants completed a total of 30 pseudo-randomly ordered trials per Block. Within each block, each character/nonword pair appeared once as a cued recall target and twice as a distractor. 
The full experiment consisted of six such Blocks; thus participants were exposed to each character / nonword pair a total of 18 times, and were asked to recall each pair six times. Participants were given a short practice session before the experiment commenced, and a short break after each Block.

During the recall phase, eye movements were recorded via a remote eye-tracker (TOBII X60; $60 \mathrm{~Hz}$ sampling rate), placed approximately $90 \mathrm{~cm}$ from the participant's eyes.

\section{Analytical approach for the paired associates learning task}

The three on-screen locations where the target and distractor characters had appeared during the encoding phase of each trial subsequently served as regions of interest (RoIs) for fixations during the recall phase. During this recall phase, an array of three dots replaced the characters on the screen, and participants attempted to recall the corresponding nonword target. The midpoint of each RoI occupied the same region of the screen as the original character. For any given trial, we could thus determine the proportion of fixations to a given RoI (e.g., the former location of the target character) relative to those elsewhere on screen.

Errors in the paired associate learning task were operationally defined as any nonword productions that did not fully concord with the correct CVC nonword. Analyses apply confirmatory logistic mixed effects regression, via the glmer::binomial function in the lme4 v1.12 library (Bates, Maechler, Bolker \& Walker, 2016) in R v3.3.2 (R Development Core Team, 2016). The model includes four fixed effects, plus their interactions: 1.) ReaderType $\{$ typical $=-0.5$, dyslexic $=0.5\}$ is contrast-coded and centered so other parameter estimates describe main effects for both groups, and interactions with ReaderType describe differences between the groups; 2.) Block $\{\log (1: 6)$, centered $\}$ captures target repetition over the course of the experiment $\left.{ }^{1} ; 3.\right)$

\footnotetext{
1 Block is log-transformed because repetition effects typically follow a log function; accordingly, the transformation demonstrably improves model fit. This transformation
} 
FixatedAnyROI, $\{$ no $=-0.5$, yes $=0.5\}$ is contrast coded and centered, coding whether the participant fixated at least one RoI during the recall phase of the trial; and finally 4.)

PrimaryFixation, conceptually nested within FixatedAnyROI, and thus not interacting with it, is contrast coded and centered, coding whether the chance-adjusted fixations on a distractor RoI were greater than those on the target $\mathrm{RoI}^{2}$. The model also includes a maximal random effects structure (Barr, Levy, Scheepers, \& Tily, 2013), omitting correlations between random effects to facilitate convergence. $P$ value estimations use the Wald approximation method.

\section{Results}

\section{Literacy and General Cognitive Ability}

Background measures for both groups are summarized in Table 1. Consistent with their diagnoses, the dyslexic group correctly read significantly fewer words and nonwords than did the controls. Although the dyslexic group were highly accurate when reading familiar words (unsurprising for well-compensated university students), their performance showed marked deficits in word reading and naming fluency, as well as deficits in nonword accuracy and fluency_all hallmarks of adult dyslexia (Bruck, 1998; Lefly \& Pennington, 1991; Shaywitz, 2003; Shaywitz \& Shaywitz, 2008). The groups did not significantly differ on either IQ measure.

is not crucial to our findings, however, and including Block as linear predictor would not change any claimed results.

2 To adjust for chance fixation patterns, distractor fixation rates were halved before computing PrimaryFixation. 
Table 1: Group scores on background measures. ${ }^{a}$ Words per minute. ${ }^{b}$ Raw scores in seconds. ${ }^{c} \mathrm{~T}$ scores. $* p<.05, * * p<.01, * * * p<.001$.

\begin{tabular}{|c|c|c|c|c|c|}
\hline \multirow[b]{2}{*}{ Source } & \multirow[b]{2}{*}{ Measure } & \multicolumn{2}{|c|}{ Mean (SD) } & \multirow[b]{2}{*}{$t$} & \multirow[b]{2}{*}{ Cohen's $d$} \\
\hline & & $\begin{array}{l}\text { Dyslexic } \\
\mathrm{N}=20\end{array}$ & $\begin{array}{l}\text { Typical } \\
\mathrm{N}=20\end{array}$ & & \\
\hline \multirow[t]{4}{*}{ TOWRE } & Word reading acc. \% & $97(3.15)$ & $98(1.41)$ & $2.55^{*}$ & -.41 \\
\hline & Word reading rate ${ }^{a}$ & $96(28.88)$ & $121(22.45)$ & $2.98 * *$ & -.96 \\
\hline & Nonword reading acc. $\%$ & $78(13.84)$ & $93(4.04)$ & $4.77 * * *$ & -1.47 \\
\hline & Nonword reading rate ${ }^{\mathrm{a}}$ & $57(18.91)$ & $82(17.58)$ & $4.40 * * *$ & -1.37 \\
\hline СТОРP & RAN $^{\mathrm{b}}$ & $17.03(4.51)$ & $12.74(2.32)$ & $3.56 * * *$ & 1.19 \\
\hline \multirow[t]{2}{*}{ WASI } & Verbal-IQ $^{\mathrm{c}}$ & $\begin{array}{l}44.84 \\
(12.45)\end{array}$ & $45.0(7.52)$ & 0.04 & -0.01 \\
\hline & Nonverbal-IQ $^{\mathrm{c}}$ & $40.61(2.75)$ & $39.45(2.23)$ & 0.57 & -0.49 \\
\hline
\end{tabular}

\section{Accuracy and fixation behaviour: General characteristics}

Excluding 423 trials (5.9\%) where eye tracking was lost left 6,777 trials for our fixation-based error analyses (3313 dyslexic, 3464 typical), summarised in Table 2. Both reader groups produced moderate proportions of recall errors throughout the experiment, allaying potential concerns about floor or ceiling effects. The two groups fixated target and distractor ROIs in quite similar proportions $\left(\chi^{2}(2)=1.43, p=.49\right)$, primarily fixating the target RoI in $27.9 \%$ of trials, one of the two distractor ROIs in $39.1 \%$ (i.e. $19.5 \%$ per distractor), and neither in the remaining $33.0 \%$. Consistent with the characterisation of fixation distributions as binomial, in $91.3 \%$ of trials with at least one RoI fixation, the target RoI accounted for either more than $90 \%$ or less than $10 \%$ of them. Thus, from this measure dyslexic readers do not appear especially likely to fail to encode spatial information, nor do they appear to re-activate it less systematically during the process of memory retrieval. As illustrated in Figure 2, fixations to the target region of interest declined with stimulus repetition, consistent with the idea that, as the visual-phonological memory representation becomes stronger, recalling it becomes less strongly associated with recalling its location. 
Table 2. Summary of trial counts and subject-weighted mean error rates and response times for trials considered in the fixation analyses. Not listed: 16 trials with fixations split equally between the target and distractor RoIs.

\begin{tabular}{|c|c|c|c|c|c|c|c|c|c|}
\hline & \multicolumn{3}{|c|}{ No RoI fixations } & \multicolumn{3}{|c|}{ Primarily fixated target } & \multicolumn{3}{|c|}{ Primarily fixated distractor } \\
\hline & Trials & Error rate & Mean RT & Trials & Error rate & Mean RT & Trials & Error rate & Mean RT \\
\hline Typical & $\begin{array}{l}1157 \\
(33.5 \%) \\
\end{array}$ & $\begin{array}{l}231 \\
(17.1 \%) \\
\end{array}$ & $859 \pm 22 \mathrm{~ms}$ & $\begin{array}{l}944 \\
(27.3 \%)\end{array}$ & $\begin{array}{l}230 \\
(26.1 \%)\end{array}$ & $1130 \pm 38 \mathrm{~ms}$ & $\begin{array}{l}1354 \\
(39.5 \%)\end{array}$ & $\begin{array}{l}321 \\
(24.2 \%) \\
\end{array}$ & $1119 \pm 32 \mathrm{~ms}$ \\
\hline Dyslexic & $\begin{array}{l}1073 \\
(32.5 \%)\end{array}$ & $\begin{array}{l}396 \\
(31.5 \%)\end{array}$ & $1079 \pm 33 \mathrm{~ms}$ & $\begin{array}{l}943 \\
(28.5 \%)\end{array}$ & $\begin{array}{l}332 \\
(37.5 \%)\end{array}$ & $1369 \pm 46 \mathrm{~ms}$ & $\begin{array}{l}1290 \\
(39.0 \%)\end{array}$ & $\begin{array}{l}576 \\
(44.9 \%)\end{array}$ & $1384 \pm 39 \mathrm{~ms}$ \\
\hline
\end{tabular}

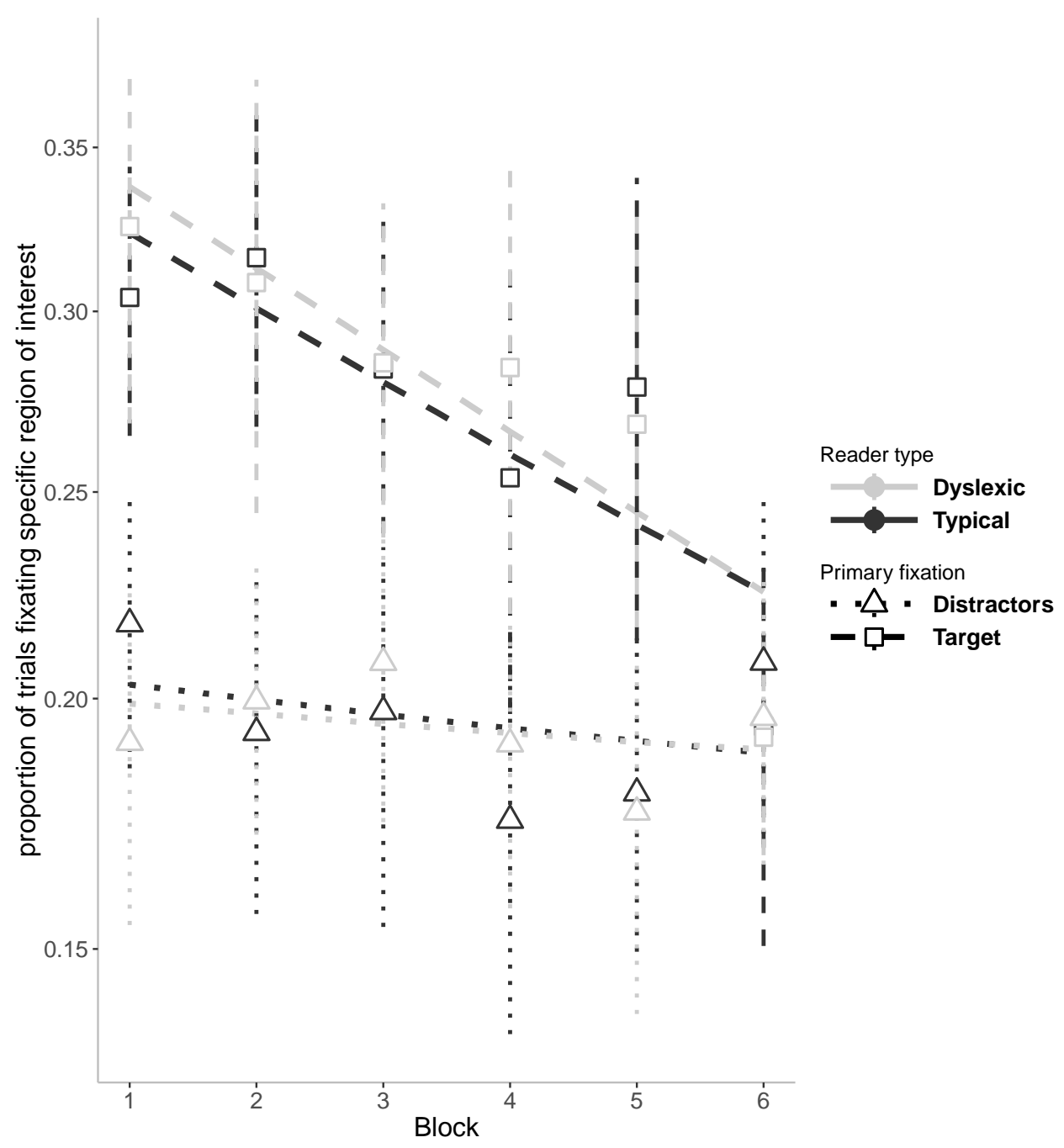

Figure 2. Mean proportion of trials in each block where a participant fixated the former location of either the target or one of the two distractors (total distractor trials divided by two), plotted on a logit scale. Lines depict logistic regression model fits. Error bars represent bootstrapped confidence intervals. 
Error patterns as a function of looking-at-nothing: Part I

As described in the Method section, we used logistic mixed effects regression, reported in Table 3, to consider error rates as a function of reading impairment (ReaderType), target repetition $(\log ($ Block)$)$, and eye fixation patterns (FixatedAnyROI) indicating whether the participant fixated at least one RoI during the recall phase of the trial, and PrimaryFixation, indicating which RoI the participant fixated most. As illustrated in Figure 3a, dyslexic participants erred more than twice as often as typical readers (odds ratio: $2.36: 1 ; \beta_{\text {ReaderType }}=.86, S E=.24, p<.001$ ). Although participants generally benefitted from stimulus repetition, erring less in later blocks (odds ratio: $\left.0.54: 1 ; \beta_{\log (\text { Block })}=-.62, S E=.07, p<.001\right)$, a marginal interaction suggests that dyslexic participants improved less than typical readers (odds ratio: $1.25: 1 ; \beta_{\text {ReaderType } x \log (\text { Block) }}=.22, S E=$ $.12, p=.07)$. This attenuated improvement thus links impaired short-term memory with impaired learning, reflecting a persistent visual-phonological binding deficit that could plausibly contribute to difficulties learning similarly arbitrary letter-to-sound mappings. 

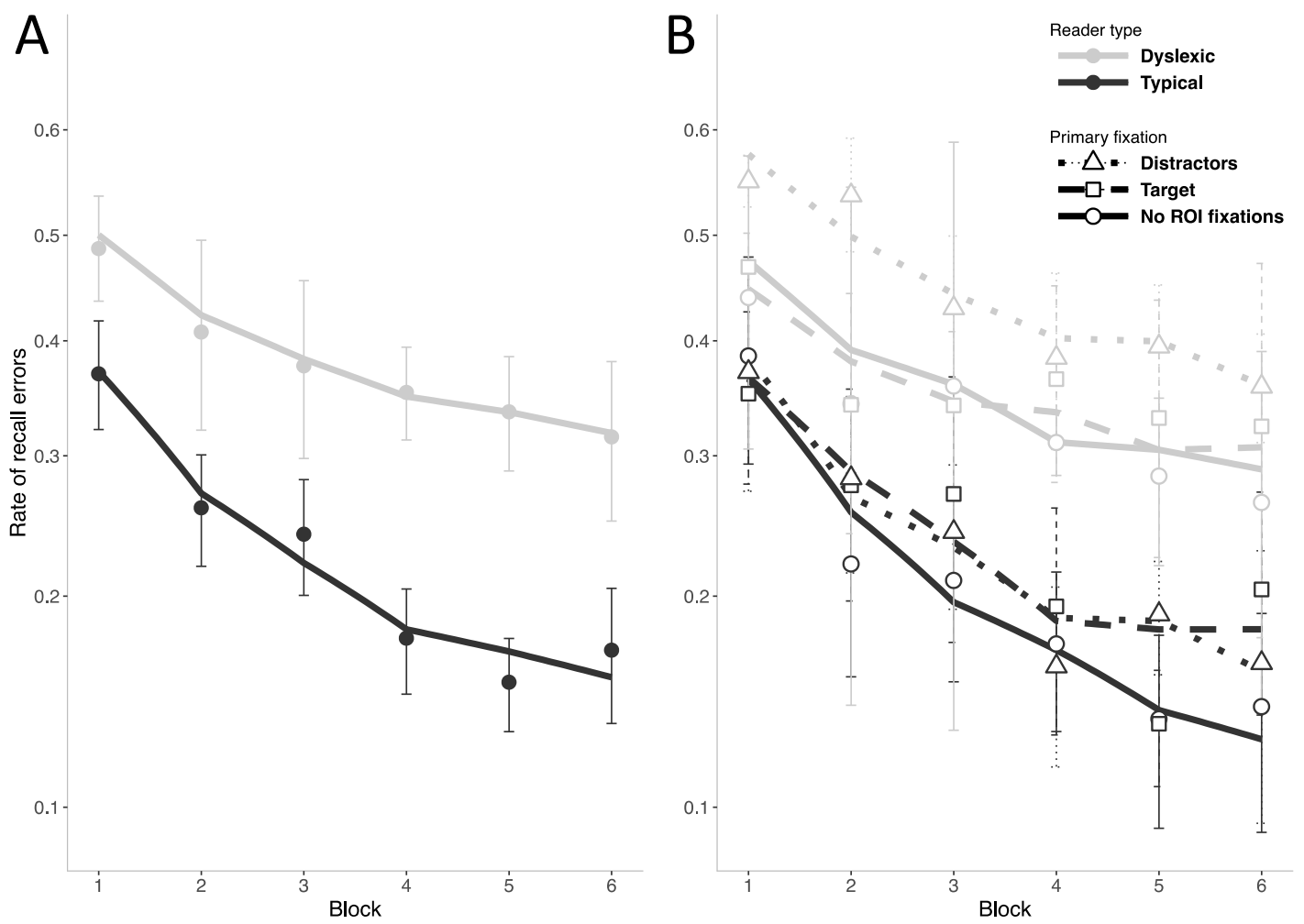

Figure 3: Subject-weighted mean recall error rates as a function of repetition (i.e. Block) and reading ability. Panel (a) depicts overall error rates for dyslexic and typical readers. Panel (b) depicts the same data, broken down according to within-trial fixation behavior. Logit-scaled axis to match logistic regression error analyses. Lines depict logistic regression model fits (Table 3). Error bars represent bootstrapped confidence intervals.

Table 3: Summary of a logistic mixed effects regression analysis of cued recall error frequency, as a function of dyslexia (ReaderType), repetition $(\log ($ Block $))$, and the existence and location of looks-at-nothing within the trial (FixatedAnyRoI and PrimaryFixation, respectively).

\begin{tabular}{lllll}
\hline & Coef $\beta$ & $\mathrm{SE}(\beta)$ & $\mathbf{p}$ & $\mathrm{OR}(\exp (\beta))$ \\
\hline (Intercept) & -0.96 & 0.12 & $<.001$ & 0.38 \\
ReaderType (typical, dyslexic) & 0.86 & 0.24 & $\mathbf{< . 0 0 1}$ & 2.36 \\
$\log$ (Block) & -0.62 & 0.07 & $\mathbf{< . 0 0 1}$ & 0.54 \\
ReaderType:log(Block) & 0.22 & 0.12 & $\mathbf{0 . 0 7}$ & 1.25 \\
FixatedAnyROI (no, yes) & 0.27 & 0.08 & $\mathbf{< . 0 0 1}$ & 1.32 \\
PrimaryFixation(target, distractor) & 0.17 & 0.1 & $\mathbf{0 . 0 8 9}$ & 1.19 \\
ReaderType:FixatedAnyROI & 0.01 & 0.16 & 0.93 & 1.01 \\
ReaderType:PrimaryFixation & 0.51 & 0.21 & $\mathbf{0 . 0 1 5}$ & 1.66 \\
log(Block):FixatedAnyROI & 0.05 & 0.11 & 0.61 & 1.06 \\
$\log$ (Block):PrimaryFixation & -0.07 & 0.12 & 0.57 & 0.93 \\
ReaderType:log(Block):FixatedAnyROI & -0.14 & 0.21 & 0.51 & 0.87 \\
ReaderType:log(Block):PrimaryFixation & -0.15 & 0.26 & 0.56 & 0.86 \\
\hline
\end{tabular}


Considering the idea that RoI fixations might serve as retrieval cues, we can now ask whether RoI fixations might generally be associated with more accurate recall. As illustrated in Figure 3b, participants actually made more errors on trials where they had fixated the former stimulus locations than when they did not (odds ratio: $1.32: 1 ; \beta_{\text {FixatedAnyROI }}=.27, S E=.08, p<.001$ ), an overall trend that did not significantly differ between reader groups (odds ratio: 1.01:1; $\beta_{\text {ReaderTypex }}$ FixatedAnyROI $=.01, S E=.16, p=.93)$. As mentioned in the Introduction, although this pattern may seem inconsistent with general idea that looks-to-nothing reflect retrieval success, it may be explained by more generally considering looks-at-nothing as reflecting retrieval effort. The consistency of the trend across groups would therefore imply that dyslexic and typical readers are similarly able to deploy visual attention in cases of more effortful retrieval.

If fixating former stimulus locations is generally associated with less accurate recall, does it matter specifically which location a participant fixates? Although an overall trend suggests that fixating the former location of a distractor is generally associated with worse recall than fixating the former location of a target (odds ratio: $1.19: 1 ; \beta_{\text {PrimaryFixation }}=.17, S E=.10, p=.089$ ), a significant interaction indicates that this association is stronger for (and perhaps exclusive to) dyslexic participants (odds ratio: $1.66: 1 ; \beta_{\text {ReaderType } x \text { PrimaryFixation }}=.51, S E=.21, p=.015$; see also Figure $3 b$ ). Thus, although dyslexic and typical readers fixate former stimulus locations similarly often, even distributing their fixations to similar locations (recall Table 2), fixation locations are more strongly associated with accurate memory retrieval for the participants with dyslexia. No other error effects approach significance (all $p<.50$ ).

\section{Response times as a function of looking-at-nothing}

The error analyses indicate not only that dyslexic participants recalled novel visualphonological bindings less accurately overall, but also that their recall accuracy was more strongly associated with the specific on-screen locations that they fixated. Whereas typical readers' looking- 
at-nothing behaviours were associated with higher error rates regardless of which RoI they fixated, dyslexic readers' recall was specifically less accurate on trials when they fixated the former location of a distractor. Does this difference imply that dyslexic readers use location information differently? If so, we might broadly expect the differences in error patterns to be accompanied by differences in response times (although predicting specific differences would require a detailed generative model).

To examine this general possibility, we follow-up the error analysis by considering the same set of predictors in a linear mixed effects regression of log-transformed response times (Table 4; Figure 4). Thus, the RT model includes the same predictors, interactions, and maximal random effects structure as that for the errors. To adjust for possible differences in the preparations of errors versus correct responses, we add to the model ErrorOutcome $\{-.5, .5\}$ and its two- and threeway interactions with the preexisting predictors. ${ }^{3}$ This analysis excludes, as probable voicekey errors, $278(4.1 \%)$ response times of less than 350ms; $p$-value estimations again use the Wald approximation method.

Table 4: Summary of a linear mixed effects regression analysis of log-transformed cued recall latencies, as a function of response error (ErrorOutcome), dyslexia (ReaderType), repetition $(\log ($ Block $))$, and the existence and location of looks-at-nothing within the trial (FixatedAnyRoI and PrimaryFixation, respectively). Millisecond effect estimates are generated by back-transforming the effect at the intercept.

${ }^{3}$ Although this approach produces a model that is somewhat overparameterised for the dataset, the same set of significant effects emerges, with similar magnitudes, if applying a model-building approach instead. 


\begin{tabular}{lrrlr}
\hline & Coef $\beta$ & SE $(\beta)$ & $p$ & in ms \\
\hline (Intercept) & 6.916 & 0.031 & - & 1008.3 \\
ReaderType (typical, dyslexic) & 0.133 & 0.061 & $\mathbf{0 . 0 3}$ & 134.0 \\
$\log ($ Block) & -0.109 & 0.023 & $<.001$ & -110.0 \\
ReaderType:log(Block) & -0.030 & 0.045 & 0.51 & -30.0 \\
FixatedAnyROI (no, yes) & 0.186 & 0.023 & $<.001$ & 188.0 \\
PrimaryFixation (target, distractor) & -0.015 & 0.015 & 0.31 & -15.0 \\
ReaderType:FixatedAnyROI & -0.004 & 0.048 & 0.93 & -4.0 \\
ReaderType:PrimaryFixation & -0.002 & 0.033 & 0.96 & -2.0 \\
log(Block):FixatedAnyRoI & -0.038 & 0.025 & 0.12 & -38.0 \\
$\log ($ Block):PrimaryFixation & -0.022 & 0.028 & 0.42 & -22.0 \\
ReaderType:log(Block):FixatedAnyROI & 0.065 & 0.049 & 0.19 & 66.0 \\
ReaderType:log(Block):PrimaryFixation & -0.031 & 0.048 & 0.53 & -31.0 \\
ErrorOutcome (no, yes) & 0.254 & 0.018 & $<.001$ & 257.0 \\
ErrorOutcome:log(Block) & 0.009 & 0.026 & 0.73 & 9.0 \\
ErrorOutcome:FixatedAnyRoI & 0.103 & 0.029 & $<.001$ & 104.0 \\
ErrorOutcome:PrimaryFixation & 0.027 & 0.033 & 0.42 & 27.0 \\
ErrorOutcome:ReaderType & 0.008 & 0.035 & 0.81 & 8.0 \\
ErrorOutcome:ReaderType:log(Block) & -0.004 & 0.052 & 0.93 & -4.0 \\
ErrorOutcome:log(Block):FixatedAnyRoI & 0.027 & 0.045 & 0.55 & 27.0 \\
ErrorOutcome:log(Block):PrimaryFixation & 0.013 & 0.052 & 0.81 & 13.0 \\
ErrorOutcome:ReaderType:FixatedAnyROI & -0.061 & 0.059 & 0.3 & -62.0 \\
ErrorOutcome:ReaderType:PrimaryFixation & -0.079 & 0.083 & 0.34 & -80.0 \\
\hline
\end{tabular}

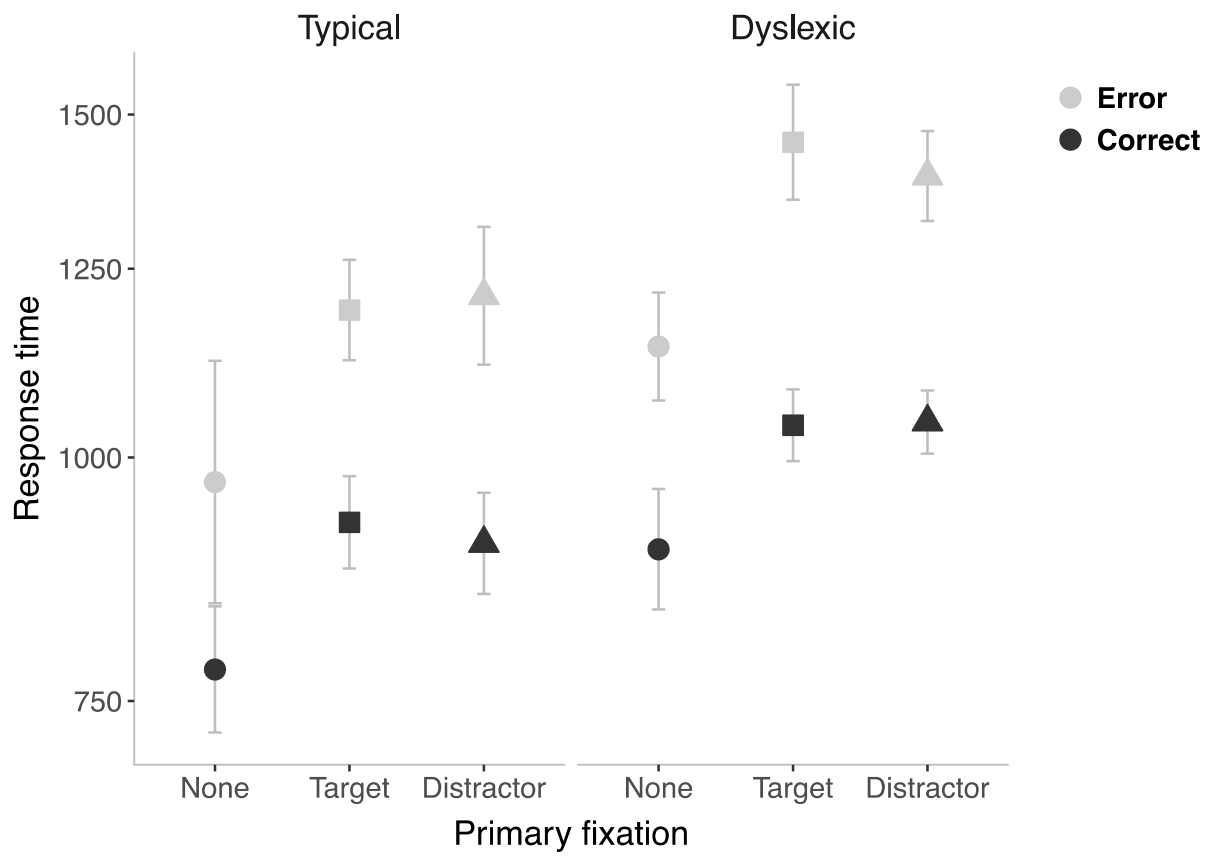

Figure 4: Log-transformed response times as a function of primary fixation, reader type, and response accuracy; for clarity, we do not depict effects of repetition. Error bars represent bootstrapped confidence intervals. 
Although dyslexic participants responded more slowly than typical participants in general $\left(135 \mathrm{~ms} ; \beta_{\text {ReaderType }}=0.133, p=.030\right)$, no interactions with dyslexia approach significance (all $\left.p>.1\right)$. Participants generally responded faster with repetition $\left(-110 \mathrm{~ms} ; \beta_{\log (\text { Block })}=-0.109, p=<.001\right)$, echoing the repetition priming observed in the error analysis. ${ }^{4}$ As suggested by the summary data in Table 2, participants were, overall, substantially slower to respond on trials when they had fixated former stimulus locations $\left(188 \mathrm{~ms} ; \beta_{\text {FixatedAnyRoI }}=0.186, p<.001\right)$, again echoing the pattern in the error analysis and consistent with the idea that looking-at-nothing behavior reflects instances of more effortful retrieval. Erroneous responses were also generally slower than correct responses $\left(257 \mathrm{~ms} ; \beta_{\text {ErrorOutcome }}=0.254, p<.001\right)$, an effect that was more pronounced in trials where the participant had fixated the former location of a stimulus $\left(104 \mathrm{~ms} ; \beta_{\text {ErrorOutcome } x \text { FixatedAnyRoI }}=0.103, p\right.$ $<.001) .{ }^{5}$ Finally, in contrast to an error pattern wherein dyslexics' responses were more likely than typicals' to coincide with their fixation locations, there was little evidence of such a trend in the response times: there was no indication that fixating a former distractor location was associated with especially slow responses in general $\left(-15 \mathrm{~ms} ; \beta_{\text {PrimaryFixation }}=-.015, S E=.15, p=.30\right)$, nor with slower correct responses or faster errors in particular $\left(27 \mathrm{~ms} ; \beta_{\text {ErrorOutcome } x \text { PrimaryFixation }}=.027, S E=\right.$ $.33, p=.42)$, and there was little evidence that dyslexic participants might differ in this respect (-

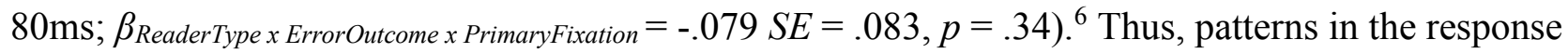
latencies echoed the error patterns in most respects, except that dyslexics' target location fixations

\footnotetext{
${ }^{4}$ Two nonsignificant interactions $\left(\beta_{\log (\text { Block }) \text { x FixatedAnyRoI }}=-0.038, p=.12 \beta_{\log (\text { Block }) x}\right.$ FixatedAnyRoI $x$ ReaderType $=0.65, p=.19$ ) simply reflect relatively stable response times in trials where typical readers responded without fixating any region of interest. ${ }^{5}$ A substantial but nonsignificant trend $\left(-62 \mathrm{~ms} ; \beta_{\text {ReaderType } x \text { ErrorOutcome } x \text { FixatedAnyRoI }}=-.61, p\right.$ $=.30$ ) suggests that this interaction was less pronounced for dyslexic participants, largely because even their correct no-fixation responses were relatively slow. ${ }^{6}$ Though obviously far from significant, this three-way interaction is the kind of response time manifestation that we might expect if looks to the former target location specifically facilitated dyslexic participants' correct response selections, and looks to other RoIs inhibited them (e.g. instead speeding the selection of a non-target response).
} 
were not reliably associated with faster target retrieval, as might have been predicted by a corresponding error effect.

Given dyslexics' slower responses, perhaps looks-at-nothing had more time bias their response selection? If so, we might expect stronger fixation-contingent error effects in verbal responses when there was a longer delay between the initial fixation and the verbal response. To assess this possibility, we calculated eye-voice spans for each trial, plotted in Figure 5. However, verbal accuracy was not further modulated by eye-voice span for either group, suggesting at least that the act of implementing a target-relevant eye movement did not feed-back to influence verbal recall (cf Staudte \& Altmann, 2017), and thus that the difference between dyslexic and typical response patterns was not simply a matter of timing.

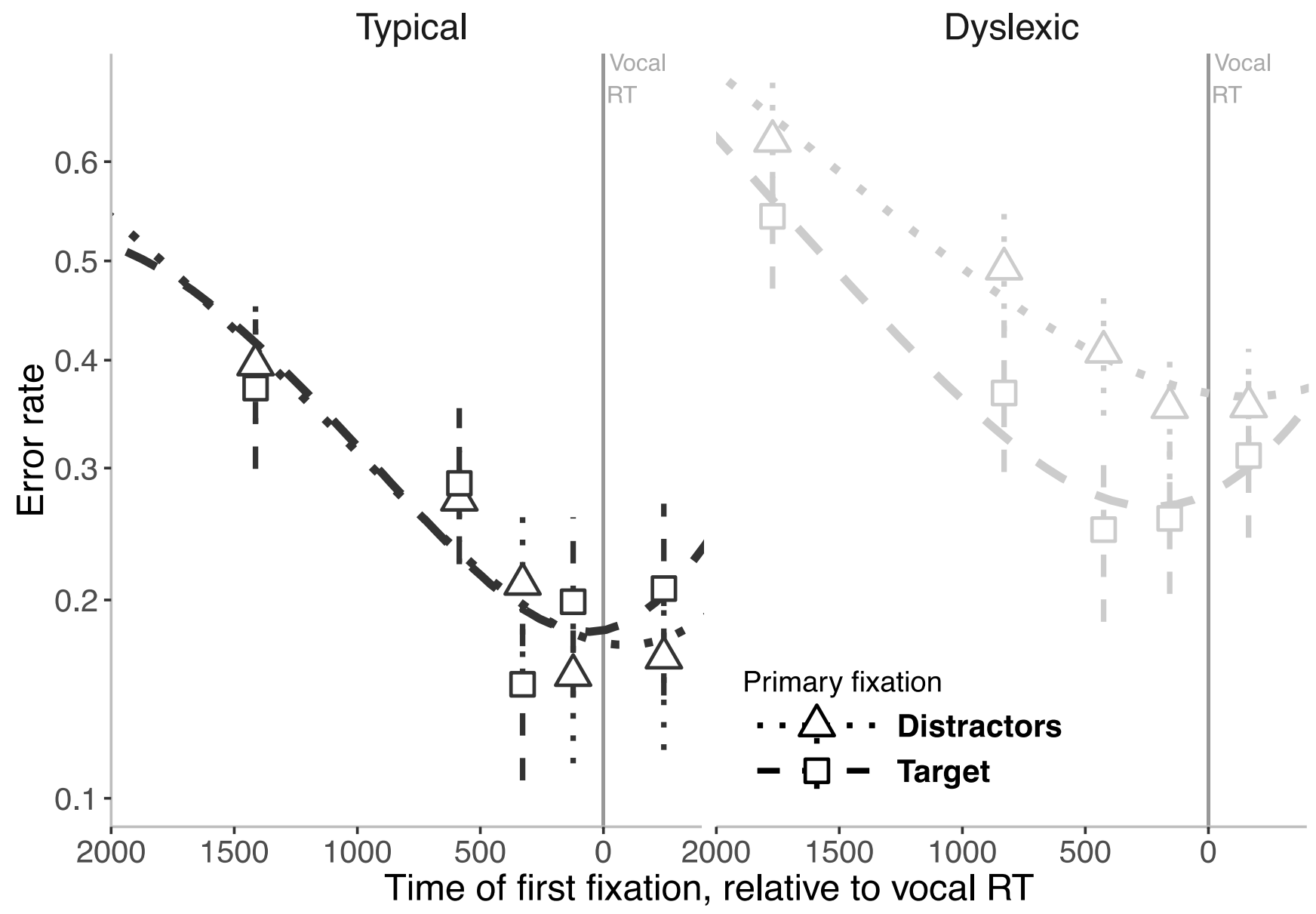


Figure 5: Error rate as a function of primary fixation, and eye-voice span (i.e. the onset time of the first RoI fixation, relative to the onset of the vocal response), for typical and dyslexic readers. Lines depict three-degree polynomials, fitted to individual data points. Error bars represent bootstrapped confidence intervals.

\section{Error patterns as a function of looking-at-nothing: Part II}

Results of the first fixation analysis indicated that recent episodic details played a stronger role in dyslexics' visual-phonological recall than typical readers'. We now turn to the question of statistical learning, by considering the role of location consistency across multiple trials. For this analysis, we distinguished between trials where the target appeared in the same location as it had previously - which we term 1-back consistent trials $^{7}$ (Figure 6, Panel A)- from those where it appeared in a different location - 1-back inconsistent trials (Figure 6, Panel B). Such 1-back consistent trials constituted about a third of all trials, 1-back inconsistent the remainder ${ }^{8}$. To the extent that participants retain and access durable representations of stimulus locations, fixation locations should modulate accuracy for the 1-back consistent trials more than for the 1-back inconsistent. The analysis revealed that typical participants' target RoI fixations were specifically associated with more accurate responses for 1-back consistent trials than 1-back inconsistent trials (odds ratio: 0.52:1; $\beta_{\text {ReaderType } x \text { PrimaryFixation } x \text { 1BackConsistency }}=-.66, S E=.32, p=.04$; Table 5; also cf. Figure 6 Panels A and B), supporting the idea that typical readers do in fact benefit from looks to former target locations, but their superior memory for such former locations allows them to better use location information from multiple instances to support target stimulus retrieval. Dyslexic readers' stronger boost from fixating a target's most recent location, including the fact that their

\footnotetext{
${ }^{7}$ Note that the 1-back term here refers only to trials where the target stimulus occurred. Trials containing the same stimulus were actually separated by 1-20 other trials. 8 The first appearance of each target fit neither criterion and was thus omitted from this analysis.
} 
recall accuracy is less strongly moderated by fixating a target's previous locations, may therefore be associated with their less-robust retention of that information over multiple trials.
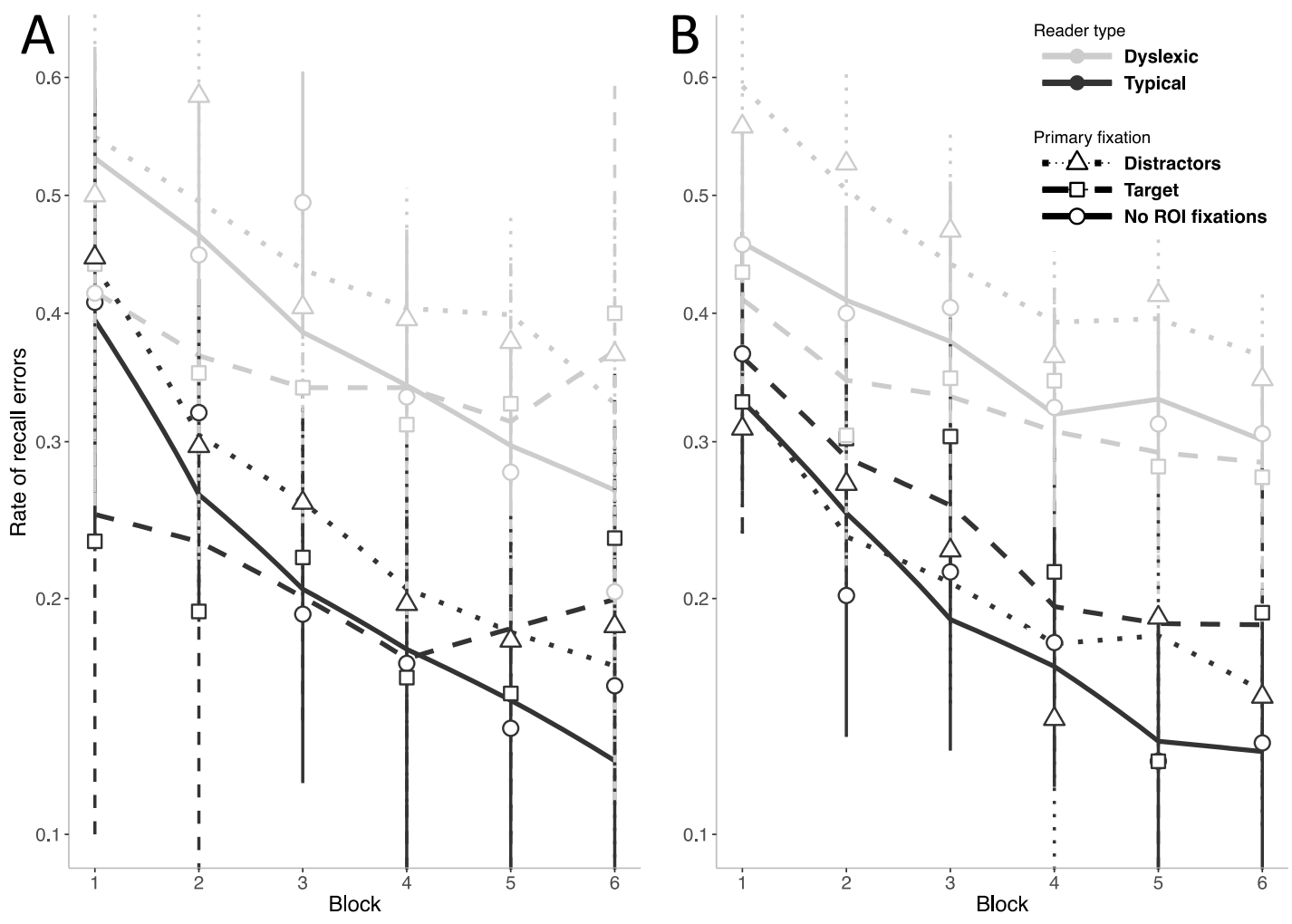

Figure 6: Error rate as a function of primary fixation and block. Panel $A=1$-back consistent trials and Panel B =1-back inconsistent trials. Lines depict logistic regression model fits (Table 5). Error bars represent bootstrapped confidence intervals.

Table 5: Summary of a logistic mixed effects regression analysis of cued recall error frequency, adding 1-back target location consistency (1BackConsistent) and its interactions to the model presented in Table 3. 


\begin{tabular}{lllll}
\hline & Coef $\beta$ & $\mathrm{SE}(\beta)$ & $\mathbf{p}$ & $\mathrm{OR}(\exp (\beta))$ \\
\hline (Intercept) & -0.99 & 0.13 & $<.001$ & 0.37 \\
ReaderType (typical, dyslexic) & 0.87 & 0.25 & $<. \mathbf{0 0 1}$ & 2.38 \\
$\log ($ Block) & -0.56 & 0.07 & $<.001$ & 0.57 \\
ReaderType:log(Block) & 0.21 & 0.14 & 0.13 & 1.23 \\
FixatedAnyROI (no, yes) & 0.28 & 0.09 & $\mathbf{0 . 0 0 1}$ & 1.33 \\
PrimaryFixation (target, distractor) & 0.19 & 0.1 & $\mathbf{0 . 0 4 9}$ & 1.21 \\
ReaderType:FixatedAnyROI & 0.07 & 0.17 & 0.66 & 1.08 \\
ReaderType:PrimaryFixation & 0.54 & 0.19 & $\mathbf{0 . 0 0 6}$ & 1.71 \\
log(Block):FixatedAnyROI & 0.07 & 0.12 & 0.57 & 1.07 \\
log(Block):PrimaryFixation & -0.18 & 0.13 & 0.18 & 0.84 \\
ReaderType:log(Block):FixatedAnyROI & -0.2 & 0.25 & 0.42 & 0.82 \\
ReaderType:log(Block):PrimaryFixation & -0.12 & 0.3 & 0.68 & 0.88 \\
1BackConsistent (no, yes) & 0.06 & 0.07 & 0.37 & 1.06 \\
1BackConsistent:ReaderType & -0.04 & 0.15 & 0.8 & 0.96 \\
1BackConsistent:log(Block) & -0.06 & 0.13 & 0.62 & 0.94 \\
1BackConsistent:ReaderType:log(Block) & 0.03 & 0.24 & 0.89 & 1.03 \\
1BackConsistent:FixatedAnyROI & -0.05 & 0.14 & 0.74 & 0.96 \\
1BackConsistent:PrimaryFixation & 0.2 & 0.16 & 0.19 & 1.23 \\
1BackConsistent:ReaderType:FixatedAnyROI & 0.15 & 0.28 & 0.59 & 1.16 \\
1BackConsistent:ReaderType:PrimaryFixation & -0.66 & 0.32 & $\mathbf{0 . 0 4}$ & 0.52 \\
1BackConsistent:log(Block):FixatedAnyROI & 0.33 & 0.26 & 0.21 & 1.39 \\
1BackConsistent:log(Block):PrimaryFixation & -0.2 & 0.29 & 0.5 & 0.82 \\
1BackConsistent:ReaderType:log(Block):FixatedAnyROI & 0.4 & 0.52 & 0.44 & 1.49 \\
1BackConsistent:ReaderType:log(Block):PrimaryFixation & 0.58 & 0.62 & 0.34 & 1.79 \\
\hline
\end{tabular}

\section{Discussion}

Mapping letters to sounds is a key skill in reading acquisition (Harm \& Seidenberg, 1999; Manis, Seidenberg, \& Doi, 1999; Seidenberg \& McClelland, 1989). In this study we considered the mechanism underpinning this ability in adult typical readers, and sources of difficulty in dyslexic readers, as both groups learned new visual-phonological bindings over repeated exposures. We were particularly concerned with the ways in which our reading groups' verbal recall was affected by 1) episodic memory of the most recent instance of a stimulus pair (i.e., memory for items in the current trial), and 2) statistical regularities in the episodic details across repeated instances of a 
stimulus pair (i.e., learning items across multiple exposures). Thus, our primary aim was to examine the transition of a single episode memory to statistical learning of visual-phonological bindings.

Looking at nothing provided a means of assessing the role of episodic detail, because spatial information was not actually required for the visual-phonological mapping. Whereas previous investigations of looking-at-nothing behaviour have demonstrated greater accuracy when correctly fixating a target's former location, relative to a non-fixation baseline, our paradigm demonstrated for the first time that fixating a competitor's former location is also associated with worse accuracy. Thus spatial recall may be considered part of a general pattern completion process, promoting target retrieval when it fits but interfering when it does not.

\section{Visual-phonological learning}

Consistent with the idea that normally developed reading involves effective paired-associate learning, typical readers demonstrated faster, more accurate verbal recall than dyslexic readers from the first exposures to these stimuli (cf. Jones et al., 2013; Messbauer \& de Jong, 2003). Whereas previous studies typically reported a single accuracy score for paired associate learning, as a culmination of multiple exposures (e.g., Hulme et al., 2007; Wang et al., 2016; Litt et al., 2013; Litt \& Nation, 2014), here we mapped the learning process as it unfolded over a larger number of repetitions, from initial exposure through to trials in which pairs were relatively familiar (15- 18 exposures), thus better approximating the development of the stable visual-phonological bindings that form the basis of successful reading. Verbal recall accuracy data showed that typical readers also improved more quickly over multiple exposures than was the case for dyslexic readers, possibly foreshadowing dyslexic readers' general resistance to developing highly automatized letter-sound correspondences in reading (Froyen et al., 2009, 2011; Jones et al., 2016). 


\section{Visual-phonological learning as a function of recent episodic recall}

The error and response time data suggest a distinction between two general response patterns. In the first pattern, representing approximately one-third of all trials ${ }^{9}$, participants recalled the cued phonological sequence quickly and accurately without detectably fixating the former location of any target or distractor. In these retrievals, we suggest that looks-to-nothing fail to emerge because activating the episodic memory of a specific item presentation is too weak or too brief to drive the execution of eye movements to previously salient screen locations: In these cases, mapping from the orthographic input to a phonological output is relatively direct. (Although competition between locations could also theoretically prevent fixating any ROI in particular, we would also have expected it to be associated with fixations to multiple locations, a pattern that was actually quite rare in this dataset.) Such fixation-less recall moreover became more frequent with repeated exposures to visual-verbal pairs, consistent with previous findings in which looks-at-nothing decreased as memory representations became more established (e.g., Scholz et al., 2016). In the second pattern, representing the remaining two-thirds of trials, memory retrieval appears slower, less accurate, and more dependent on activation of episodic detail. Retrieval in these cases invokes more looks-to-nothing and possibly uses their planning and/or execution as ancillary cues for memory retrieval. Thus, it may be possible to situate both patterns within the kind of autoassociative network illustrated in Figure 7.

\footnotetext{
${ }^{9}$ Note that the current design employed a rather conservative assessment of looks at nothing, given presentation of the visual probe at center screen, possibly overriding original spatial location encoding, and the possibility of fixating non-ROI screen locations (contra previous studies, e.g., Scholtz et al., 2016; Wantz, Martarelli, \& Mast, 2016). Whilst we consider this an advantage for the current objectives, we note that it also comprises a potential methodological issue in its comparability to previously-used paradigms.
} 

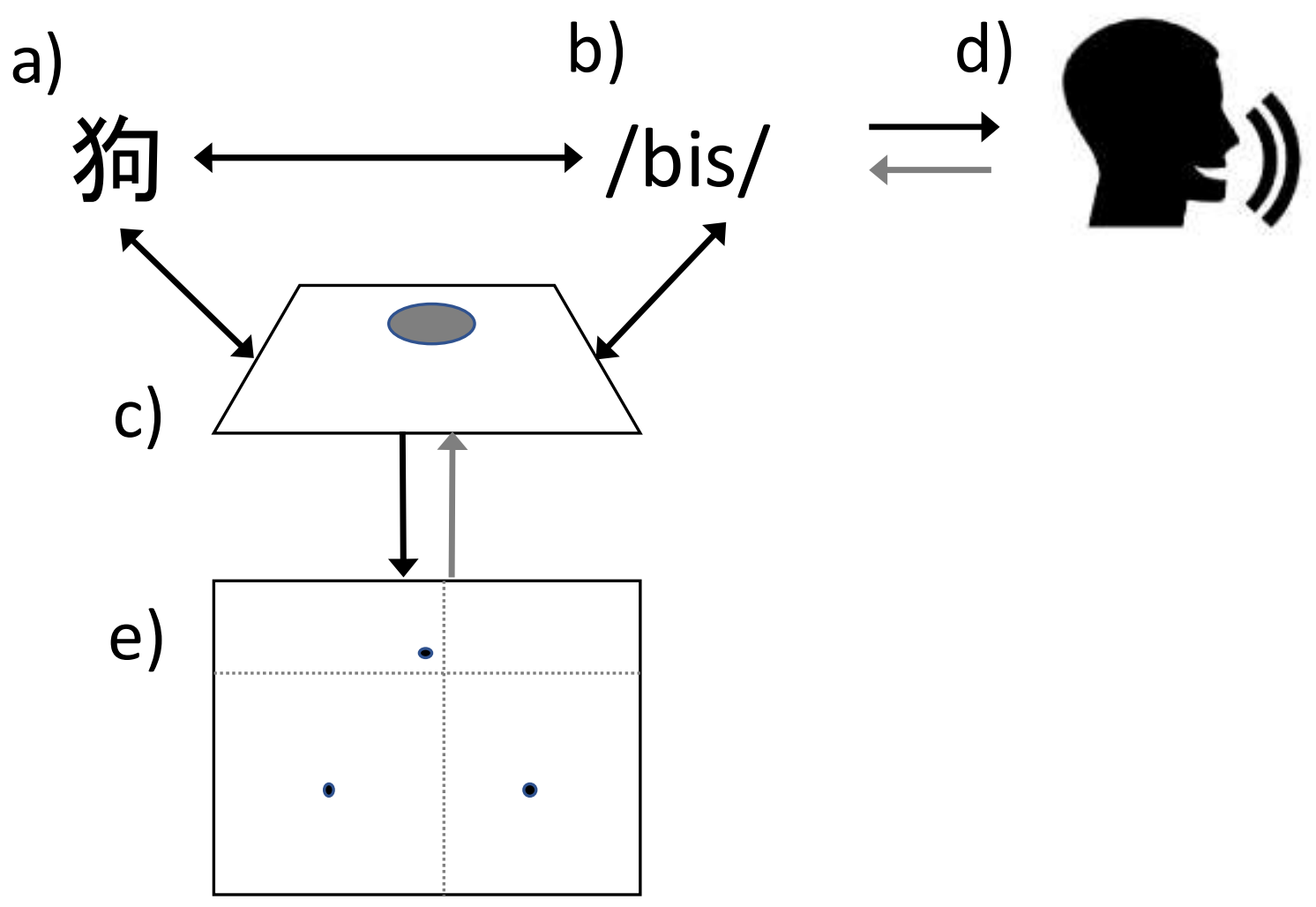

Figure 7. Schematic model of visual-phonological recall as pattern completion within an associative network. Representations of the visual symbol (a), phonological form (b), and spatial position (c) form the core network. Sufficient activation of a phonological representation drives its oral production (d), and sufficient activation of a spatial position drives oculomotor movements to it (e); planning or implementing either movement may feedback to reinforce activation of its associated representation.

Within this structure, we start with the assumption that querying a visual form in episodic memory generally spreads activation to all components of the representation - in a sense initiating retrieval via both a direct symbol-to-sound pathway and less direct symbol-to-spatial-to-sound pathway_-but observable looks-to-nothing require exceeding some activation threshold before being implemented. Looks-to-nothing may not, therefore, occur if retrieval — the sufficient activation of a single phonological form - is accomplished before that threshold is exceeded, for instance when the direct pathway is relatively strong or the spatial pathway is relatively weak. That is, whatever activation reaches the spatial pathway must be strong enough and last long enough to initiate a look-to-nothing, and such observable behaviour may therefore index 1.) the 
strength of activation of specific episodic detail (else it would be insufficient to drive an eye movement), and 2.) the weakness of activation in the direct retrieval pathway (else it would not allow sufficient time to plan and implement a movement before the response occurs).

Given that activation in the spatial pathway is sufficient to drive an RoI fixation, there is a question of how much the activation within that pathway will contribute to response selection. This pathway may be slower by virtue of being indirect - for instance, if the action of the eye movement provides a retrieval cue (Scholtz et al., 2016; reflected in a feedback connection from (e) to (c) in Figure 7), then its contribution could only begin sometime after the implementation of the movement - so it would seem reasonable to assume that information from the spatial pathway should have more time to contribute to responses that are selected later. Our data in fact provide mixed support for these initial assumptions: trials containing looks to nothing tended to be slower than those without, likely reflecting more effortful retrieval and thus consistent with recent claims that looks at nothing are associated with weaker representations (Scholz et al., 2011; Wantz et al., 2016). On the other hand, our eye-voice span analyses provided no evidence that slower responses increased the spatial pathway's contribution to response accuracy, limiting our confidence in the hypothesised feedback connection that might allow oculomotor movements to influence phonological retrieval.

Within this model, how might the role of episodic details in dyslexic readers compare to that in typical readers? Our previous findings (Jones et al, 2013) suggested that dyslexic readers were perhaps less able than typical readers to leverage spatial information to support verbal recall. In the current study, however, dyslexic participants' recall accuracy was moderated more than typical participants' by fixating a target's most recent location, showing that they do in fact encode and retrieve location information, and their verbal recall accuracy is linked to accurate spatial/episodic recall (though their verbal recall may not benefit from it as much as typical readers'). Typical participants' lack of benefit from accurate looks-at-nothing was also prima facie inconsistent with 
much previous research. Before we can account for this pattern of results, it is first necessary to describe the findings relating to participants' visual-phonological learning in the context of repeated exposures to stimulus pairs.

\section{Visual-phonological learning as a function of multiple instances}

One remarkable feature of this paradigm was its stimulus repetition: to approximate readers' acquisition of stable orthographic-to-phonological mappings, each visual-verbal pair appeared 18 times over the course of the experiment. Participants generally grew more accurate each time they recalled a visual-phonological binding, suggesting access to and/or integration of multiple episodic memory traces. Typical readers' accuracy benefitted marginally more from repetition, which could therefore indicate that they either retain and access past traces more effectively, or better integrate them, e.g. forming the kind of composite representations that might be more useful for quick and effortless access (such as position-independent representation of letters for reading novel strings). Indeed, our looking-at-nothing data also revealed that typical readers considered a target's prior locations to a significantly greater degree than did dyslexic readers: whereas dyslexic readers' phonological recall was better when fixating the target's most recent location, and worse when fixating a distractor's most recent location, for typical readers this modulation only occurred when location became a more consistent feature of the target (i.e. when the stimulus had appeared in the same location at least twice in a row). Considering single-trial and multi-trial analyses together, one possible interpretation is that dyslexic readers responses primarily derived from memory of the most recent episode alone, whereas typical readers' responses better integrated multiple episodes to create more robust prototype or exemplar-like concept representations.

At least three related processes in concept learning may contribute to typical readers' greater success and stronger modulation by location consistency. First, in an exemplar (or instance) view of concept learning (e.g. Logan, 2002; Medin \& Schaffer, 1978), people are expected to retain 
details of each instance of a concept - such as the location of symbol-sound binding - and use them to evaluate concept properties on demand; from this perspective, typical readers may be better able than dyslexic readers to incorporate multiple instances, due to either encoding or access. If dyslexic readers' impaired implicit memory led to increased noise during the observation of a current event (as suggested by Jaffe-Dax et al., 2015), that could provide the basis for such an encoding deficit. Failing to adequately encode previous instances could leave dyslexic readers' verbal recall more dependent on retrieval cues that are readily available in short-term memory, such as a stimulus' most recent location (but by the same token, erroneously attending to a competing location could be sufficient to derail the fragile recall process). Second, skilled reading may benefit from a process of distilling multiple instances of the same symbol-sound binding into a more integrated representation that abstracts away extraneous details; this kind of progressive abstraction might be accomplished by an incremental learning algorithm that learns through experience to emphasise consistent distinctive features and ignore inconsistent and non-distinctive features (e.g. Krushke, 1992; Love et al., 2004; see Altmann, 2017, for a recent review). Because symbol-sound bindings were neither consistently bound with particular locations in this experiment, nor modulated by them (i.e. the same symbol was paired with the same sound regardless of its location), location represents one such extraneous detail, so it is possible that typical readers are better at this type of, essentially, incremental prototype formation. Finally, recent category learning models also describe a possibility of de-emphasising less-useful features of input via temporary changes in attention (e.g. ibid). If typical readers learn not to attend to single instances of location-because it often miscues alternative responses - then dyslexic readers' persistent attention to this lower-quality predictor could be associated with other aspects of attentional control, such as their greater difficulty ignoring visual and auditory distractors (e.g., Bouma \& Legein, 1977; Callens, Whitney, Tops, \& Brysbaert, 2013; Sperling, Lu, \& Manis, 2005, 2006). 


\section{Conclusion}

Learning to read involves shifting from deliberate episodic recall of letter identities to automatic multiple-constraint satisfaction. Identifying the most relevant features of orthophonological concepts, and tracking other features of potential value, are important aspects of this transition. Recall-time phenomena such as 'looks to nothing' reveal readers' inclusion of spatial location as part of their initial episodic representations, and more generally their consideration of contexts as potentially valid cues to orthophonological mappings, which they statistically evaluate through repetition. Readers with dyslexia are initially less able to recall orthophonological bindings, and benefit less from repetition than those without, due in part to their difficulty tracking such contexts and perhaps more general difficulty integrating such memories over multiple instances.

Acknowledgements: This work was supported by the Coleg Cymraeg Cenedlaethol [MWJ; www.colegcymraeg.ac.uk]. We thank Patricia Bestelmeyer and Joshua Payne for insightful comments on an earlier version of this manuscript. We also thank Roger Johansson and one other anonymous reviewer for their helpful comments and suggestions.

\section{References}

Ahissar, M. (2008). Dyslexia and the anchoring-deficit hypothesis. Trends in cognitive sciences, 11, 458-465.

Altmann, G. T. (2017). Abstraction and generalization in statistical learning: implications for the relationship between semantic types and episodic tokens. Phil. Trans. R. Soc. B, $372,20160060$. 
Altmann, G. T. (2004). Language-mediated eye movements in the absence of a visual world: The 'blank screen paradigm'. Cognition, 93, B79-B87.

Baddeley, A. D., Allen, R. J., \& Hitch, G. J. (2011). Binding in visual working memory: The role of the episodic buffer. Neuropsychologia, 49, 1393-1400.

Barr, D. J., Levy, R., Scheepers, C., \& Tily, H. J. (2013). Random effects structure for confirmatory hypothesis testing: Keep it maximal. Journal of Memory and Language, $68,255-278$.

Bates, D., Maechler, M., Bolker, B., \& Walker, S. (2016). lme4: Linear mixed-effects models using Eigen and S4. 2014. R package version 1.1-7.

Blau, V., van Atteveldt, N., Ekkebus, M., Goebel, R., \& Blomert, L. (2009). Reduced neural migration of letters and speech sounds links phonological and reading deficits in adult dyslexia. Current Biology, 19, 503-508. doi:10.1016/j.cub.2009.01.065

Bouma, H., \& Legein, C. P. (1977). Foveal and parafoveal recognition of letters and words by dyslexics and by average readers. Neuropsychologia, 15, 69-80.

Brockmole, J. R., \& Franconeri, S. L. (Eds.). (2009). Binding: A special issue of visual cognition. Hove, UK: Psychology Press.

Bruck, M. (1998). Outcomes of adults with childhood histories of dyslexia. Reading and spelling: Development and disorders, 179, 200.

Callens, M., Whitney, C., Tops, W., \& Brysbaert, M. (2013). No deficiency in left-to-right processing of words in dyslexia but evidence for enhanced visual crowding. The Quarterly Journal of Experimental Psychology, 66, 1803-1817.

Denckla, M. B., \& Rudel, R. G. (1976). Rapid 'automatized'naming (RAN): Dyslexia differentiated from other learning disabilities. Neuropsychologia, 14, 471-479.

Ehri, L. C. (2005). Learning to read words: Theory, findings, and issues. Scientific Studies of reading, $9,167-188$. 
Ehri, L. C., \& Saltmarsh, J. (1995). Beginning readers outperform older disabled readers in learning to read words by sight. Reading and Writing, 7, 295-326.

Ferreira, F., Apel, J., \& Henderson, J. M. (2008). Taking a new look at looking at nothing. Trends in cognitive sciences, 12, 405-410.

Froyen, D. J., Bonte, M. L., van Atteveldt, N., \& Blomert, L. (2009). The long road to automation: neurocognitive development of letter-speech sound processing. Journal of Cognitive Neuroscience, 21, 567-580.

Froyen, D., Willems, G., \& Blomert, L. (2011). Evidence for a specific cross-modal association deficit in dyslexia: An electrophysiological study of letter-speech sound processing. Developmental science, 14, 635-648.

Harm, M. W., \& Seidenberg, M. S. (1999). Phonology, reading acquisition, and dyslexia: Insights from connectionist models. Psychological Review, 106, 491-528. doi:10.1037/0033-295X.106.3.491

Hintzman, D. L. (1986). " Schema abstraction" in a multiple-trace memory model. Psychological review, 93, 411.

Hommel, B., \& Colzato, L. S. (2009). When an object is more than a binding of its features: Evidence for two mechanisms of visual feature integration. Visual Cognition, 17, 120 -140. doi:10.1080/1350628080234978

Hoover, M. A., \& Richardson, D. C. (2008). When facts go down the rabbit hole: Contrasting features and objecthood as indexes to memory. Cognition, 108, 533-542. doi:10.1016/j.cognition.2008.02.011.

Hulme, C., Goetz, K., Gooch, D., Adams, J., \& Snowling, M. (2007). Paired-associate learning, phoneme awareness and learning to read. Journal of Experimental Child Psychology, 96, 150-166. doi:10.1016/j.jecp.2006.09.00 
Jaffe-Dax, S., Frenkel, O., \& Ahissar, M. (2017). Dyslexics' faster decay of implicit memory for sounds and words is manifested in their shorter neural adaptation. Elife, 6 .

Jaffe-Dax, S., Lieder, I., Biron, T., \& Ahissar, M. (2016). Dyslexics' usage of visual priors is impaired. Journal of vision, 16, 10-10.

Jaffe-Dax, S., Raviv, O., Jacoby, N., Loewenstein, Y., \& Ahissar, M. (2015). A Computational Model of Implicit Memory Captures Dyslexics' Perceptual Deficits. The Journal of Neuroscience, 35, 12116-12126.

Jahn, G., \& Braatz, J. (2014). Memory indexing of sequential symptom processing in diagnostic reasoning. Cognitive Psychology, 68, 59-97. doi:10.1016/j.cogpsych.2013.11.002.

Johansson, R., Holsanova, J., Dewhurst, R., \& Holmqvist, K. (2012). Eye movements during scene recollection have a functional role, but they are not reinstatements of those produced during encoding. Journal of Experimental Psychology: Human Perception and Performance, 38, 1289-1314. doi:10.1037/a0026585.

Johansson, R., \& Johansson, M. (2014). Look here, eye movements play a functional role in memory retrieval. Psychological Science, 25, 236-242. doi:10.1177/0956797613498260.

Jones, M. W., Ashby, J., \& Branigan, H. P. (2013). Dyslexia and fluency: parafoveal and foveal influences on rapid automatized naming. Journal of Experimental Psychology: Human Perception and Performance, 39, 554.

Jones, M. W., Branigan, H. P., Parra, M. A., \& Logie, R. H. (2013). Cross-modal binding in developmental dyslexia. Journal of Experimental Psychology: Learning, Memory, and Cognition, 39, 1807. 
Jones, M. W., Kuipers, J. R., \& Thierry, G. (2016). ERPs reveal the time-course of aberrant visual-phonological binding in developmental dyslexia. Frontiers in Human Neuroscience, 10.

Jones, M. W., Obregón, M., Kelly, M. L., \& Branigan, H. P. (2008). Elucidating the component processes involved in dyslexic and non-dyslexic reading fluency: An eyetracking study. Cognition, 109, 389-407.

Kruschke, J. K. (1992). ALCOVE: An exemplar-based connectionist model of category learning. Psychological Review, 99, 22-44.

LaBerge, D., \& Samuels, S. J. (1974). Toward a theory of automatic information processing in reading. Cognitive psychology, 6, 293-323.

Laeng, B., Bloem, I. M., D’Ascenzo, S., \& Tommasi, L. (2014). Scrutinizing visual images: The role of gaze in mental imagery and memory. Cognition, 131, 263-283. doi:10.1016/j.cognition.2014.01.003.

Lefly, D. L., \& Pennington, B. F. (1991). Spelling errors and reading fluency in compensated adult dyslexics. Annals of dyslexia, 41, 141-162.

Lervåg, A., \& Hulme, C. (2010). Predicting the growth of early spelling skills: Are there heterogeneous developmental trajectories?. Scientific Studies of Reading, 14, 485513.

Litt, R. A., de Jong, P. F., van Bergen, E., \& Nation, K. (2013). Dissociating crossmodal and verbal demands in paired associate learning (paired-associate learning): What drives the paired-associate learning-reading relationship? Journal of Experimental Child Psychology, 115, 137-149.

Litt, R. A., \& Nation, K. (2014). The nature and specificity of paired associate learning deficits in children with dyslexia. Journal of Memory and Language, 71, 71-88. 
Logan, G. D. (2002). An instance theory of attention and memory. Psychological Review, 109, 376-400. http://doi.org/10.1037//0033-295X.109.2.376

Logie, R. H., Brockmole, J., \& Jaswal, S. (2011). Feature binding in visual working memory is unaffected by task-irrelevant changes of location, shape and color. Memory and Cognition, 39, 24-36.

Love, B. C., Medin, D. L., \& Gureckis, T. M. (2004). SUSTAIN: a network model of category learning. Psychological Review, 111, 309-32. http://doi.org/10.1037/0033295X.111.2.309

Manis, F. R., Seidenberg, M. S., \& Doi, L. M. (1999). See Dick RAN: Rapid naming and the longitudinal prediction of reading subskills in first and second graders. Scientific Studies of Reading, 3, 129-157.doi:10.1207/s1532799xssr0302_3

Medin, D. L., \& Schaffer, M. M. (1978). Context theory of classification learning. Psychological review, 85, 207.

McClelland, J. L., McNaughton, B. L., \& O'reilly, R. C. (1995). Why there are complementary learning systems in the hippocampus and neocortex: insights from the successes and failures of connectionist models of learning and memory. Psychological review, 102, 419.

Medin, D. L., \& Schaffer, M. M. (1978). Context theory of classification learning. Psychological Review, 85, 207-238.

Messbauer, V. C., \& de Jong, P. F. (2003). Word, nonword, and visual paired associate learning in Dutch dyslexic children. Journal of Experimental Child Psychology, 84, 77-96.

Nosofsky, R. M. (1986). Attention, similarity, and the identification-categorization relationship. Journal of experimental psychology: General, 115, 39. 
Richardson, D. C., \& Spivey, M. J. (2000). Representation, space and Hollywood Squares: Looking at things that aren't there anymore. Cognition, 76, 269-295.

Scholz, A., Mehlhorn, K., \& Krems, J. F. (2016). Listen up, eye movements play a role in verbal memory retrieval. Psychological Research, 80, 149-158.

Shaywitz, S. E. (2003). Overcoming dyslexia: A new and complete science-based program for reading problems at any level. Knopf.

Shaywitz, S. E., \& Shaywitz, B. A. (2008). Paying attention to reading: The neurobiology of reading and dyslexia. Development and Psychopathology, 20, 1329-1349.

Seidenberg, M. (2017). Language at the Speed of Sight: How we Read, Why so Many CanÕt, and what can be done about it. Basic Books.

Seidenberg, M. S., \& McClelland, J. L. (1989). A distributed, developmental model of word recognition and naming. Psychological Review, 96, 523-568. doi:10.1037/0033295X.96.4.523.

Snowling, M. J. (2000). Dyslexia (2nd edn). Malden, MA: Blackwell Publishing.

Sperling, A. J., Lu, Z. L., Manis, F. R., \& Seidenberg, M. S. (2005). Deficits in perceptual noise exclusion in developmental dyslexia. Nature Neuroscience, 8, 862-863.

Sperling, A. J., Lu, Z. L., Manis, F. R., \& Seidenberg, M. S. (2006). Motion-Perception Deficits and Reading Impairment It's the Noise, Not the Motion. Psychological Science, 17, 1047-1053.

Staudte, M., \& Altmann, G. T. (2017). Recalling what was where when seeing nothing there. Psychonomic Bulletin \& Review, 24, 400-407.

Torgesen, J.K., Wagner, R.K., \& Rashotte, C. A. (1999). Test of Word Reading

Efficiency (TOWRE). Austin, Texas: Pro-Ed.

Treisman, A. (2006). Object tokens, binding and visual memory. In H. Zimmer, A. Mecklinger, \& U. Lindenberger (Eds.), Handbook of binding and memory: 
Perspectives from cognitive neuroscience (pp. 315-338). New York, NY: Oxford University Press.

Treisman, A., \& Gelade, G. (1980). A feature integration theory of attention. Cognitive Psychology, 12, 97-136. doi:10.1016/0010-0285(80)90005-5

Treisman, A., \& Zhang, W. (2006). Location and binding in visual working memory. Memory and Cognition, 34, 1704 -1719. doi:10.3758/BF03195932.

Vanrullen, R. (2009). Binding hardwired versus on-demand feature conjunctions. Visual Cognition, 17, 103-119.

Vellutino, F. R., Steger, J. A., Harding, C. J., \& Phillips, F. (1975). Verbal vs non-verbal paired-associates learning in poor and normal readers. Neuropsychologia, 13, 75-82.

Vellutino, F. R., Scanlon, D. M., \& Spearing, D. (1995). Semantic and phonological coding in poor and normal readers. Journal of Experimental Child Psychology, 59, 76-123.

Wagner, R. K., Torgesen, J. K., \&Rashotte, C. A. (1999). Comprehensive Test of Phonological Processing. Austin, TX: PRO-Ed.

Wang, S., Allen, R. J., Lee, J. R., \& Hsieh, C. E. (2015). Evaluating the developmental trajectory of the episodic buffer component of working memory and its relation to word recognition in children. Journal of Experimental Child Psychology, 133, 16-28.

Wang, H. C., Wass, M., \& Castles, A. (2016). Paired-Associate Learning Ability Accounts for Unique Variance in Orthographic Learning. Scientific Studies of Reading, 471482.

Wantz, A. L., Martarelli, C. S., \& Mast, F. W. (2016). When looking back to nothing goes back to nothing. Cognitive processing, 17, 105-114.

Warmington, M., \& Hulme, C. (2012). Phoneme awareness, visual-verbal paired-associate learning, and rapid automatized naming as predictors of individual differences in reading ability. Scientific Studies of Reading, 16, 45-62. 
Wechsler, D. (1999). Wechsler Abbreviated Scale of Intelligence (WASI). San Antonio, TX: Harcourt Assessment.

Wimmer, H. (1993). Characteristics of developmental dyslexia in a regular writing system. Applied Psycholinguistics, 14, 1-33.

Wimmer, H., Mayringer, H., \& Landerl, K. (1998). Poor reading: A deficit in skillautomatization or a phonological deficit? Scientific Studies of Reading, 2, 321-340.

Žarić, G., González, G. F., Tijms, J., van der Molen, M. W., Blomert, L., \& Bonte, M. (2015). Crossmodal deficit in dyslexic children: practice affects the neural timing of letter-speech sound integration. Frontiers in Human Neuroscience, 9. 